\title{
Cutting Tool Wearing Identification Through Predictive Maintenance and Its Impact on Surface Quality
}

\author{
Jose Maria GONZALEZ CASTRO ${ }^{\mathrm{a}, 1}$, Giselle RAMIREZ SANDOVAL ${ }^{\mathrm{b}}$, \\ Eduard VIDALES COCA ${ }^{\mathrm{b}}$, Nuri CUADRADO LAFOZ ${ }^{\mathrm{b}}$, Francesc BONADA ${ }^{\mathrm{a}}$, \\ ${ }^{a}$ Eurecat, Centre Tecnologic de Catalunya, Unit of Applied Artificial Intelligence, Av. \\ Universitat Autònoma, 23, 08290 Cerdanyola del Vallès, Spain \\ ${ }^{\mathrm{b}}$ Eurecat, Centre Tecnologic de Catalunya, Unit of Metallic and Ceramic Materials, \\ Plaça de la Ciència 2, 08243, Manresa, Spain
}

\begin{abstract}
Smart manufacturing has been in the media for a long time, but the reality shows that traditional mechanical manufacturing industries have not been able to implement data solutions aligned with Industry 4.0 standards. This work inquiries into the possibility of measuring cutting tool vibrations for $\mathrm{CNC}$ turning machines and presents the data analysis and a predictive model to identify tool wearing that can affects integrity surface quality of the manufactured component. These preliminary results are orientated towards implementing a predictive maintenance methodology in cutting tools.
\end{abstract}

Keywords. CNC turning machine, Machine Learning, Tool Wearing Prediction, Industry 4.0

\section{Introduction}

During last decades there has been a special attention in predicting surface quality through vibration measurement during the milling process. This has been carried out to introduce Industry 4.0 technologies into metal manufacturing workshops where datadriven models are not used due to the lack of measured data during manufacturing. In this field, interesting research has been devoted to develop surface quality prediction methods for the roughness quality [1] [2]. Nevertheless, these works have been developed on a controlled lab where manufacturing parameters are known and recorded. Although the research shows promising results, real production applications face with increased challenges, such as technicians availability to introducing manufacturing parameters in an external software during process planning. Industrial manufacturing has to deal with dynamic scenarios that affect parameters such as tool wearing, changes in the material batches, also the technician can modify machining parameters as feed rate or the radian and axial depth cut during manufacturing [3]. These complex scenarios present difficulties for the implementation of previously developed models for on-line surface quality prediction, especially for small workshops.

${ }^{1}$ Jose Maria Gonzalez Castro, Eurecat, Centre Tecnològic de Catalunya, Applied Artificial Intelligence Unit, Av. Universitat Autònoma, 23, 08290 Cerdanyola del Vallès, Spain; E-mail: jose.gonzalez@eurecat.org. 
The objective of the research presented in this work is to develop a predictive model that can identify surface quality degradation during manufacturing, reducing the costs of manufacturing defective components and identifying the problem when quality assurance tests are performed. AVINT project pushes forward the current surface quality prediction techniques by researching into the possibility to develop a model that only depends on on-line measured vibration data that can be correlated with tool wearing. This approach will allow to shift from current preventive maintenance strategies to change the cutting tools into a more effective and cost-saving predictive maintenance strategy where tools are used until they really need to be changed, maximizing both tool lifespan and part quality.

\section{Experimental Data and Feature Engineering}

The data schema consists of two sensors registering 4 channels. The first three channels correspond to the 3 axes from an accelerometer with a sampling frequency of $10 \mathrm{kHz}$. The fourth channel corresponds to an electro-acoustic sensor with a maximum sampling frequency of $200 \mathrm{kHz}$. These 4 channels are stored on a time-series database and organized by experiment.

A set of experiments were performed on a HAAS ST-10Y CNC lathe machine before performing industrial measurements on a real production facility. Five tools with different levels of degradation were tested. The objective was to evaluate the possibility to determine the tool degradation based only on recording the vibrations produced during operation.

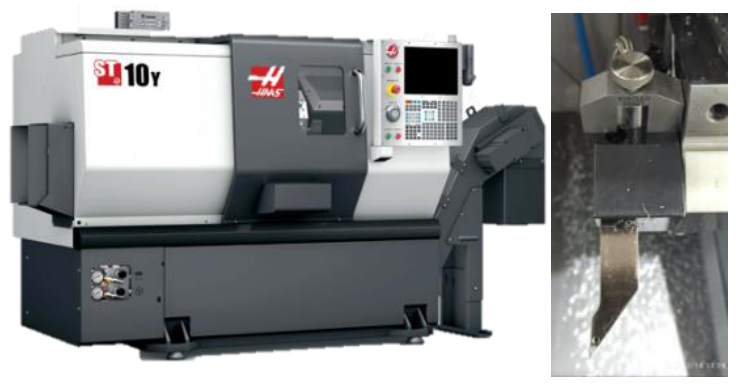

Figure 1. experimental setup

Five different tools were tested by manufacturing a cylindrical test specimen of aluminium alloy. Three cylindrical specimens were manufactured for each tool. The tools were named as e1, e2, e 3 e 4 and e5. Tools e1 and e 3 were new tools, while e2, e4 and 55 had different wear level at the cutting edge produced under lab control before the machining test (see Table 1). Worn material of tools was measured by means of a focus variation microscope obtaining $3 \mathrm{D}$ images of the cutting edges.

Table 1. Wear at the cutting edge before machining test

\begin{tabular}{cccccc}
\hline & $\mathrm{e} 1$ & $\mathrm{e} 2$ & $\mathrm{e} 3$ & $\mathrm{e} 4$ & $\mathrm{e} 5$ \\
\hline $\begin{array}{c}\text { Removed material } \\
{[\mathrm{mm} 3]}\end{array}$ & $\mathrm{New}$ & 0.017 & $\mathrm{New}$ & 0.001 & 0.019 \\
\hline
\end{tabular}

The data has been segmented to consider only periods where the selected tool is working, to obtain a region of interest to be analyzed. To extract important information from the data and to reduce the dimensionality, statistical descriptors were extracted for 
each channel, considering both time and frequency domains and enabling to reduce the sampling values to $0.1 \mathrm{~Hz}$. The time-domain descriptors consist of the mean value, standard deviation, skewness, kurtosis, maximum peak value and the dispersion of the 10 most prominent peaks on each 0.1 seconds of data. Frequency data consisted of the energy of the signal for different frequency bands based on the work described in [4].

\section{Analysis of the Experiments}

Due to the high dimensionality of the data descriptors, a manifold Multi-Dimensional Scaling (MDS) is used to visualize the data in a two-dimensional space area. MDS establish the possibility to distinguish between tools only using the data by representing the similarity contained in the data as a geometrical distance. Figure 2 shows the MDS visualization results.

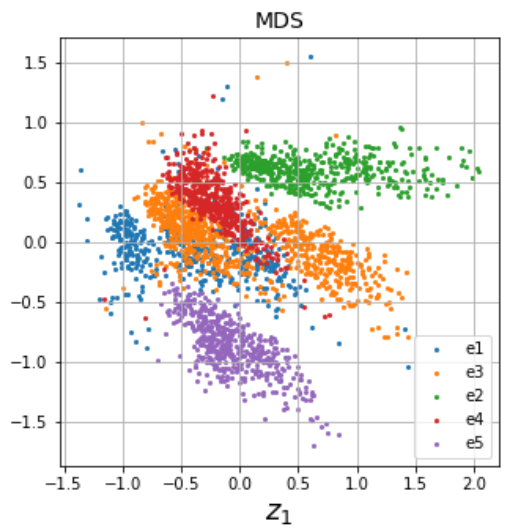

Figure 2. Result of visualizing vibration data on two dimensions through Multi-dimensional scaling (MDS).

The MDS presented in Figure 2 shows that tool set e1, e 3 and e4 contain a higher level of similarity with respect to other tools, mainly expressed in the formation of a single cluster. On the other side, tools e 2 and e 5 show their data clustered on opposite sides from the main cluster showing dissimilarity with all the other tools.

The surface quality of the manufactured component was assessed by measuring four roughness parameters (see Table 2): the arithmetical mean heigh for line (Ra) and surface (Sa), the mean peak width/average length of elements (Rsm) and the developed interfacial area ratio (Sdr)[5]. These values were measured by means of confocal microscopy technique using a Sensofar software and according to ISO 4287 and ISO 25178 .

Table 2. Surface quality of the manufactured cylindrical specimens

\begin{tabular}{ccccc}
\hline Tool & Ra $[\boldsymbol{\mu m}]$ & Sa $[\boldsymbol{\mu m}]$ & Rsm [mm] & Sdr [\%] \\
\hline e1 & $6.95 \pm 0.34$ & $6.97 \pm 0.23$ & $0.20 \pm 0.01$ & $8.17 \pm 1.53$ \\
e2 & $11.87 \pm 2.27$ & $13.63 \pm 1.68$ & $0.22 \pm 0.01$ & $62.30 \pm 4.10$ \\
e3 & $7.36 \pm 0.38$ & $7.33 \pm 0.10$ & $0.19 \pm 0.01$ & $10.65 \pm 1.07$ \\
e4 & $8.97 \pm 0.71$ & $8.97 \pm 0.27$ & $0.19 \pm 0.01$ & $17.60 \pm 0.30$ \\
e5 & $4.09 \pm 0.79$ & $4.33 \pm 0.89$ & $0.25 \pm 0.01$ & $2.80 \pm 0.60$ \\
\hline
\end{tabular}

Cutting edge of tools after manufacturing tests are shown in Figure 3. The green color shows the initial geometry, red and blue colors represent the adhered and removed material, respectively. 


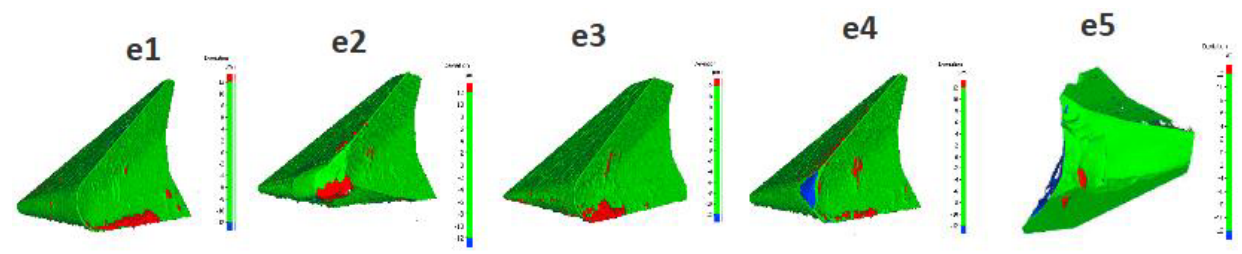

Figure 3. Cutting edge of the tools after test (images acquired by focus variation microscopy). The red color represents adhered material, blue color is the removed material from the tool, and the green color is the initial geometry of the tool. All the images are oriented to show the maximum variation of the tool with respect to the new tool. The images are scaled within -12 and +12 micrometers.

\section{Discussion and Conclusions}

Data shows that it is possible to distinguish between tools showing a similarity in the recorded data related to the surface quality and their degradation. More precisely, e1 and e3 being new tools, together with e4 which was slightly degraded produced surfaces with similar quality.

On the other side, e 2 and e5 produced divergent signals with respect to other tools. This difference is seen on the removed material which is 10 times higher with respect to e4. The sharper cutting edge of e5 tool produced by microchipping before the manufacturing test is remarkable and it probably explains the small rugosity values generated during the experiment compared with other tools.

The analysis of the experiments shows an encouraging result that it is possible to track tool wear by only monitoring tool vibrations. The project is continuing by measuring tool vibration on real production environments. Currently, industrial data is available and further analysis are going on. The nature of the problem suggests that an unsupervised method can be used to identify on-line tool degradation by means of signal variation during cutting work.

\section{Acknowledgment}

This work has been carried out in the framework of the AVINT project, which has received funding from the European Union through European funding for regional development (FEDER).

\section{References}

[1] D. Korzeniewski y N. Znojkiewicz, «The influence of vibrations on surface roughness formed during precision boring,» Archives of Mechanical Technology and Materials, vol. 37, 2017.

[2] S. Palani y U. Natarajan, «Prediction of surface roughness in CNC end milling,» International Journal of Advanced Manufacturing Technology, pp. 1033-1042, 11 June 2011.

[3] P. Benardos y G.-C. Vosniakos, «Predicting Surface Roughness in Machining: a Review,» International Journal of Machine Tools and Manufacture, vol. 43, pp. 833-844, 2003.

[4] G. Quintana, M. Garcia-Romeu y J. Ciurana, «Surface roughness monitoring application based on artificial neural networks for ball-end milling operations,» Journal of Intelligent Manufacturing, pp. 607-611, 2011.

[5] KEYENCE, «Introduction to "Roughness",» [Online]. Available: https://www.keyence.eu/ss/products/microscope/roughness/. [Last acces: 1005 2021]. 